\title{
Minimal Hepatic Encephalopathy in Indians: Psychometric Hepatic Encephalopathy Score and Inhibitory Control Test for Diagnosis and Rifaximin or Lactulose for Its Reversal
}

\author{
Vinay B. Pawar*, Ravindra G. Surude, Nikhil Sonthalia, Vinay Zanwar, Samit Jain, \\ Qais Contractor and Pravin M. Rathi
}

Department of Gastroenterology, Topiwala National Medical College and BYL Ch Hospital, Mumbai, Maharashtra, India

\begin{abstract}
Background and Aims: Psychometric hepatic encephalopathy score (PHES) is used widely for diagnosis of minimal hepatic encephalopathy (MHE). This prospective study aimed to determine the utility of the inhibitory control test (ICT) for the diagnosis of MHE. Additionally, the efficacy of rifaximin and lactulose for reversal of MHE was evaluated. Methods: A total of 180 eligible cirrhotic patients underwent testing for MHE. When PHES was $\leq-5$ and ICT lures were $\geq 14$, MHE was diagnosed. The 108 patients with MHE were randomized to three groups for treatment with either lactulose, rifaximin, or placebo. Treatment outcomes were measured at the end of 3 months. Results: The 108 patients with MHE diagnosed by PHES and/or ICT accounted for $60 \%$. The diagnosis of MHE was made by both ICT and PHES positivity in 56 patients, by abnormal ICT and normal PHES in 37 patients, and by abnormal PHES and normal ICT in 15 patients. For diagnosis of MHE, ICT had sensitivity of $78.87 \%$, specificity of $66.06 \%$ with $60.22 \%$ positive predictive value and $82.76 \%$ negative predictive value. An area under the curve value of 0.724 (95\% CI: $0.653-0.788$ ) was obtained for diagnosis of MHE. Reversal of MHE was seen in $71.42 \%, 70.27 \%$ and $11.11 \%$ of patients in the rifaximin, lactulose and placebo arms $(p<0.001)$. Rifaximin showed better tolerability compared to lactulose. Conclusions: For the diagnosis of MHE, ICT is a simple tool but has lower sensitivity and better specificity than PHES. Rifaximin is as efficacious as lactulose in the treatment of MHE and better tolerated.

Citation of this article: Pawar VB, Surude RG, Sonthalia N, Zanwar V, Jain S, Contractor Q, et al. Minimal hepatic encephalopathy in Indians: Psychometric hepatic encephalopathy score and inhibitory control test for diagnosis and rifaximin or lactulose for its reversal. J Clin Transl Hepatol 2019;7(4):304312. doi: $10.14218 /$ JCTH.2017.00037.
\end{abstract}

Keywords: Minimal hepatic encephalopathy; ICT; PHES; Indians; Lactulose; Rifaximin.

Abbreviations: CTP, Child-Turcotte-Pugh; DST, digit symbol test; HE, hepatic encephalopathy; HRQOL, health-related quality of life; ICT, inhibitory control test; LTT, line tracing test; MELD, model of end-stage liver disease; MHE, minimal hepatic encephalopathy; PHES, psychometric hepatic encephalopathy score; SDT, serial dotting test.

Received: 4 June 2017; Revised: 28 May 2018; Accepted: 14 February 2019

*Correspondence to: Vinay B. Pawar, Department of Gastroenterology, Topiwala National Medical College and BYL Ch Hospital, Dr. A.L Nair Road, Mumbai, Maharashtra 400008, India. Tel: +22-23021639, E-mail: docvinu@gmail.com
Introduction

Hepatic encephalopathy (HE) consists of a spectrum of neuropsychiatric abnormalities that are seen in patients with liver dysfunction and after exclusion of other known neurological diseases. ${ }^{1-3}$ Minimal hepatic encephalopathy (MHE), a subgroup of $\mathrm{HE}$ is a neurocognitive complication of cirrhosis. Most of the time, MHE remains undiagnosed and is diagnosed in $20-80 \%$ of patients with liver cirrhosis with the help of special diagnostic testing. ${ }^{1}$ It can progress to overt $\mathrm{HE}$ and impaired health-related quality of life (HRQOL). ${ }^{4,5}$ Furthermore, MHE is associated with driving impairment, resulting in high risk of road traffic accidents for these patients. ${ }^{6,7}$

MHE includes a specific spectrum of cognitive deficits, which mostly involve the domains of response inhibition, executive function, attention and vigilance. ${ }^{8-10}$ Psychometric tests are considered the gold standard for the diagnosis of MHE. These psychometric tests specifically assess the above-mentioned cognitive domains. ${ }^{11}$ The Psychometric Hepatic Encephalopathy Score (PHES) is composed of five tests, including the number connection test-A (NCT-A), number connection test-B (NCT-B), serial dotting test (SDT), line tracing test (LTT) and digit symbol test (DST). As such, the PHES can be used to assess a number of parameters, like motor speed, motor accuracy, concentration, attention, visual perception, visual-spatial orientation, visual construction and memory. These are related to most of the neuropsychological impairments in MHE.

There is also a computer-based test known as the Inhibitory Control Test (ICT), which was constructed for the evaluation of attention and response inhibition. It has been used previously to characterize traumatic brain injury, attention deficit disorder, and schizophrenia. ${ }^{12}$

It is an established fact that the clinical manifestations of $\mathrm{HE}$ in each stage can be reversed when managed by adequate therapeutic management. As mentioned above, MHE is currently underdiagnosed in patients with liver cirrhosis. MHE carries a high potential for progression to overt HE. Meanwhile, the current management of MHE is directed at reduction of ammonia production from the gastrointestinal tract. This can be achieved by administration of lactulose, a nonabsorbable disaccharide. MHE can also be managed with nonabsorbable antibiotic rifaximin. ${ }^{13-16}$

Randomized controlled studies and a meta-analysis ${ }^{17}$ have compared probiotics, prebiotics and a symbiotic with lactulose in the treatment of MHE. These studies have shown that in MHE reversal, lactulose is equally or more 
effective than the pre-pro-synbiotics. ${ }^{18,19}$ Rifaximin is a broad spectrum antibiotic; moreover, it is gut-selective and has minimal systemic absorption. It has a tolerability profile and good safety. The previous studies carried out on MHE patients have shown that rifaximin improves cognitive functions and HRQOL, as compared to placebo. ${ }^{13,20}$

In this study, we compared PHES and ICT for the diagnosis of MHE and assessed the efficacy of rifaximin and lactulose in reversal of the disease.

\section{Methods}

\section{Plan of research}

The study was conducted in a gastroenterology tertiary care center in Mumbai, India, from May 2015 to March 2017. The PHES and ICT were compared in the diagnosis of MHE. We also compared the efficacy of rifaximin and lactulose in the reversal of MHE. The study was approved by the Institutional Ethics Committee. Written informed consent was obtained from each participant before their enrollment.

\section{Characteristics of subjects/patients}

Patient selection (Fig. 1): A total of 250 patients, aged 18-65 years and with established diagnosis of liver cirrhosis without any overt symptoms of $\mathrm{HE}$ at the time of testing (West Haven grade 0), were recruited to the study. The diagnosis of cirrhosis was based on clinical history, serologic testing, radiologic imaging and liver histology, when available. All the patients were either hospitalized or attending the Outpatient Clinic of Gastroenterology. After the initial assessment, $180 / 250(72 \%)$ of the patients were included in the study. Exclusion criteria were: (1) history of recent alcohol intake or substance abuse in the last 12 weeks; (2) history of recent gastrointestinal tract bleeding; (3) serious infections and use of drugs affecting psychometric performance, like antidepressants and sedatives in the last 4 weeks; (4) previous transjugular intrahepatic portosystemic shunt or shunt surgery; (5) significant comorbid illness (e.g. heart, respiratory or renal failure); (6) visual or mental impairment; (7) neurologic diseases, like stroke, epilepsy, dementia, and parkinsonism; (8) hepatocellular carcinoma; (9) prior treatment exposure to lactulose or rifaximin, recent antibiotic use, or current probiotics; (10) past history of HE.

Among the 70 patients who were excluded, a history of overt HE was present in 10, history of recent alcohol consumption in 15 , recent variceal bleed in 15 , acute kidney injury in 15, and hepatocellular carcinoma in 5. Out of 180 total recruited patients, 30 were hospitalized, 22 of which had MHE (73.33\%). The reasons for hospitalization included newonset ascites, alcoholic hepatitis, and refractory ascites.

\section{Study protocol}

Neuropsychological assessment: The PHES has been validated in many populations of various nationalities, including German, Spanish, Italian, and Indian. ${ }^{20}$ It includes a battery of five tests: DST, number connection test-A, number connection test-B, LTT for time $(t)$ and error $(e)$, and serial dotting test (SDT). The PHES is calculated from the results of these five tests. The clinical significance of the PHES score has been previously evaluated in a large number of healthy volunteers, in addition to patients with liver cirrhosis with MHE. In Indian cirrhotic patients, the PHES score cutoff has been found to $\leq-5$ for abnormal designation. This cutoff can reliably be used for the diagnosis of MHE. ${ }^{20}$ In the present study, we used PHES scores of $\leq-5$ for the diagnosis of MHE.

ICT: This test consists of presentation of several letters at a specific interval rate (500-millisecond intervals). ${ }^{21}$ It is identical to the continuous performance test. $X$ and $Y$ letters are interspersed between these letters. The subject is asked to respond to every $X$ and $Y$ during the initial part of the training run, which shows the pre-potent response. In the latter part of the training run, the subject is asked only to respond when $X$ and $Y$ are alternating (also called as targets) and to inhibit responding when $X$ and $Y$ are not alternating (also called 'lures'). After the training run, 6 test runs, which last approximately 2 minutes each, are administered. The patient is presented with a total of 40 lures, 212 targets, and 1728 random letters in between. At the end of the test, the number of lures, target frequency, lure and target response rates, and target reaction times are automatically calculated. Good psychometric performance is indicated by lower lure response and higher target response.

In the initial studies in the literature, it was revealed that the most important variable is a lure threshold of $>5$ for the diagnosis of $\mathrm{MHE}^{7}$ The initial lure threshold of $>5$ was set too low for our population, mostly reflecting the high educational status of the selected control population. Indian patients whom we encounter in our tertiary care center are less familiar with computer use. Since the lure threshold needs to be adjusted according to the local population norms, we took the lure threshold of $\geq 14$ as the diagnostic cut off for diagnosis of $\mathrm{HE}^{22,23}$ In our study, we considered only lures and not weighted lures as suggested by Amodio et al. (unpublished study).

Treatment groups: In the current study, patients underwent a comprehensive physical examination and a detailed neurological examination that included the Mini Mental Status Examination. This was to rule-out any overt cognitive impairment before administering neuropsychological tests and the ICT. Only those patients with a Mini Mental Status Examination score of $>24$ were included. We also performed a battery of laboratory investigations, like complete hemogram, liver function tests, renal function tests, ultrasonography, esophagogastroduodenoscopy and other etiological tests, as and when required. The eligible patients were classified according to disease severity, determined by Child-TurcottePugh (CTP) classification and model for end-stage liver disease (MELD). Out of the 180 eligible patients who underwent neuropsychological assessment and ICT, 108 were positive for MHE by either ICT with lures $\geq 14$ or PHES score of $\leq-5$, or both. Subsequently patients were randomized to three groups for treatment with either lactulose $(30-60 \mathrm{~mL} /$ day to have 2-3 loose stools/day) or rifaximin (550 mg 2 times/day), or B-complex tablets as a placebo (2 times/day). We used the titrated dose of lactulose.

The patients were followed up in our outpatient department every 2 weeks in the first month and then on a oncemonthly schedule. At every visit, the patients were assessed for the development of overt HE. Treatment compliance was ensured by pill count (checking empty blisters during each visit), and confirmed by questioning of the patient's accompanying relatives. The treatment outcomes were evaluated at the end of 3 months in the form of improvement in PHES score and ICT findings. 
Pawar V.B. et al: MHE in cirrhotics

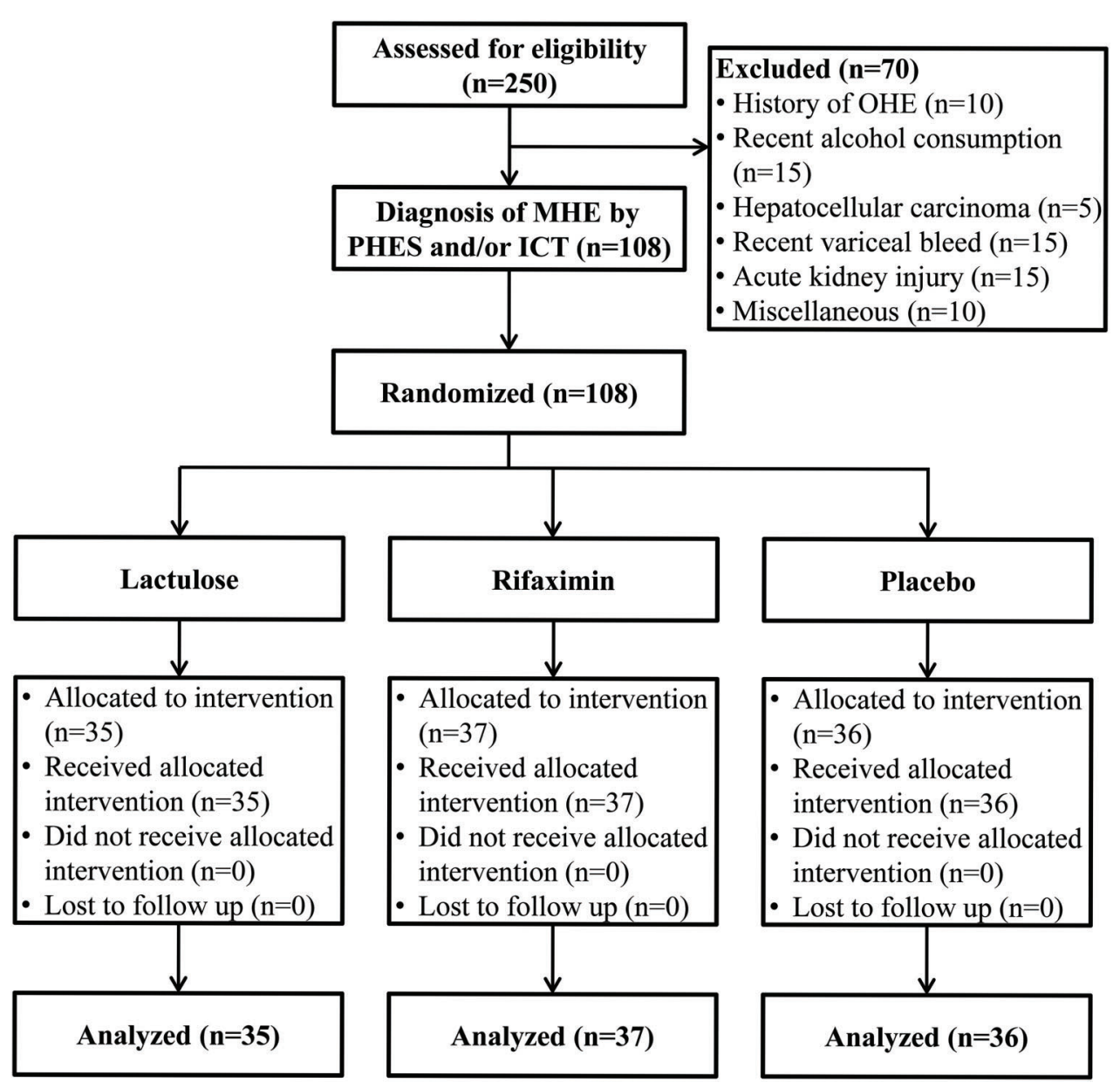

Fig. 1. Consort diagram.

Abbreviations: ICT, inhibitory control test; MHE, minimal hepatic encephalopathy; OHE, overt hepatic encephalopathy; PHES, psychometric hepatic encephalopathy score.

Outcome/end points: The primary outcome was reversal of MHE at 3 months. Reversal of MHE was defined as PHES score of $>-5$ and or ICT lures $<14$.

Sample size: Previous studies have established MHE reversal in $75.5 \%$ of patients treated with rifaximin, ${ }^{24}$ and a mean of $47.5 \%$ of those treated with lactulose, ${ }^{18}$ and $10 \%$ among those receiving no treatment. ${ }^{18}$ For power of $80 \%$, the total sample size of 105 patients (35 patients in each group) was determined as necessary to obtain accurate results.

Randomization: Patients were randomized by an independent observer according to a computer-generated list of random numbers. The study was carried out double-blinded, with both patients and investigators blinded to the study treatment.

Statistical methods: Data analysis was done by using the Statistical Package for Social Sciences version 20 (IBM Corp., Armonk, NY, USA). The chi-square test/Fischer's exact test were used to find the association between various qualitative data variables within the treatment groups and to determine the association between demographic variables with occurrence of MHE. To find the cutoff value of lures in patients with $\mathrm{MHE}$, receiver operating characteristic curves were generated. The Mann-Whitney $U$ test was used to compare cirrhotic patients with and without MHE. The Wilcoxon sign rank test was used to compare the pre- and posttreatment results of neuropsychometric tests and of ICT. A $p$-value of $<0.05$ was considered significant.

Result

A total of 250 patients with cirrhosis were evaluated for eligibility, and the 180 patients included in the study (fulfilling eligibility criteria) underwent ICT and PHES to establish the diagnosis of MHE. The clinical and personal characteristics of the patients are shown in Table 1.

Out of 180 patients, $108(60 \%)$ were diagnosed with MHE by PHES and/or ICT. Of the 108 MHE patients, 98 (90.74\%) were males. A total of 33 patients belonged to CTP class A, while 55 patients were CTP class B and 20 were CTP C. The baseline MELD score was 10 in patients, with or without MHE. Amongst the patients with $\mathrm{MHE}$, alcohol-related chronic liver disease was the most common diagnosis, being present in 63 patients, followed by hepatitis B-related cirrhosis in 18 patients.

Diagnosis of MHE was made in 56 patients by abnormal ICT and PHES, by abnormal ICT and normal PHES in 
Pawar V.B. et al: MHE in cirrhotics

Table 1. Baseline characteristics of 180 patients included in the study

\begin{tabular}{|c|c|c|c|c|}
\hline Variables & Lactulose arm & Rifaximin arm & Placebo arm & Non-MHE \\
\hline Age in years, mean \pm standard deviation & $48.4 \pm 8.23$ & $48.86 \pm 6.75$ & $48.69 \pm 7.22$ & $48.32 \pm 8.18$ \\
\hline Males, $n$ & 31 & 34 & 33 & 60 \\
\hline Education in years, median (min-max) & $10(6-13)$ & $9(6-15)$ & $10(6-15)$ & $9(6-15)$ \\
\hline \multicolumn{5}{|l|}{ Baseline CTP class } \\
\hline Class A, $n$ & 12 & 11 & 10 & 27 \\
\hline Class $\mathrm{B}, n$ & 17 & 20 & 18 & 29 \\
\hline Class $\mathrm{C}, n$ & 6 & 6 & 8 & 16 \\
\hline MELD score & $10(8-13)$ & $10(6-14)$ & $10(8-13)$ & $10(6-14)$ \\
\hline \multicolumn{5}{|l|}{ Etiology of cirrhosis } \\
\hline Alcohol, $n$ & 20 & 21 & 22 & 50 \\
\hline $\mathrm{NASH}, n$ & 3 & 5 & 5 & 6 \\
\hline Hepatitis B, $n$ & 4 & 7 & 7 & 9 \\
\hline Hepatitis C, $n$ & 2 & 3 & 3 & 4 \\
\hline $\mathrm{AIH}, n$ & 1 & 1 & 2 & 2 \\
\hline $\mathrm{BCS}, n$ & 1 & 0 & 1 & 1 \\
\hline
\end{tabular}

Abbreviations: AIH, autoimmune hepatitis; BCS, Budd-Chiari syndrome; CTP, Child-Turcotte-Pugh; MELD, model for end-stage liver disease; NASH, nonalcoholic steatohepatitis.

37 patients, and by abnormal PHES and normal ICT in 15 patients. Sensitivity and specificity of PHES was $65.7 \%$ and $100 \%$ with cutoff value for PHES of $\leq-5$. An area under the curve value of 0.841 (95\% CI: $0.779-0.891$ ) was obtained for diagnosis of MHE by PHES. Sensitivity and specificity of ICT was $78.87 \%$ and $66.06 \%$ for the diagnosis of $\mathrm{MHE}$, with $60.22 \%$ positive predictive value and $82.76 \%$ negative predictive value. An area under the curve value of 0.724 (95\% CI: 0.653-0.788) was obtained for diagnosis of MHE (Fig. 2).

The ICT lures were significantly higher (Table 2) in cirrhotic patients with MHE $(17.48 \pm 2.62 ; 95 \% \mathrm{CI}$ for mean difference: 6.87-8.15), than for those without MHE at baseline (10.01 $\pm 2.01)(p<0.001)$. The presence of ICT targets was significantly lower in cirrhotic patients with MHE (176.54 $\pm 6.67 ; 95 \% \mathrm{CI}$ for mean difference: $-19.54-16.46)$, than for those without MHE $(194.54 \pm 3.32)(p<0.001)$. The mean values (standard deviation) for NCT-A, NCT-B, SDT, LTT, and DST were 92.29 (14.75), 106.84 (24.60), 90.47 (14.83), 103.31(20.88), and 16.47 (9.58) respectively in patients with MHE and were significantly higher compared to patients without MHE (for which the values were 72.88 (5.99), 79.43 (8.62), 75.67 (8.01), 82.75 (9.12), and 18.07 (3.20) respectively; $p<0.001$ ) (Table 3 ).

Table 4 shows results of the neuropsychiatric tests and ICT in patients with MHE before and after treatment (Figs. 3 and 4 ). In the lactulose arm, MHE was present by abnormal ICT in 30 patients, out of which 23 showed reversals, and by abnormal PHES in 23 patients, out of which 14 showed reversal. Reversal of MHE was seen in 25/35 patients in the lactulose arm. In the rifaximin arm, MHE was present by abnormal ICT in 31 patients, out of which 21 showed reversal, and by abnormal PHES in 24 patients, out of which 19 showed reversal. Reversal of MHE was seen in 26/37 patients in the rifaximin arm. In the placebo group, MHE was present by abnormal ICT in 32 patients, out of which 4 had reversal, and by abnormal PHES in 24 patients, out of which 2 showed reversal. Reversal of MHE was seen in 4/36 patients in the placebo arm. Reversal of MHE was seen in $71.42 \%$ of patients in the lactulose arm, $70.27 \%$ of patients in the rifaximin arm, and $11.11 \%$ of patients in the placebo arm $(p<0.001)$. For the diagnosis of MHE by ICT, we considered only lures and not the weighted lures. Though the lures improved in patients with placebo, the improvement was

\section{Lures}

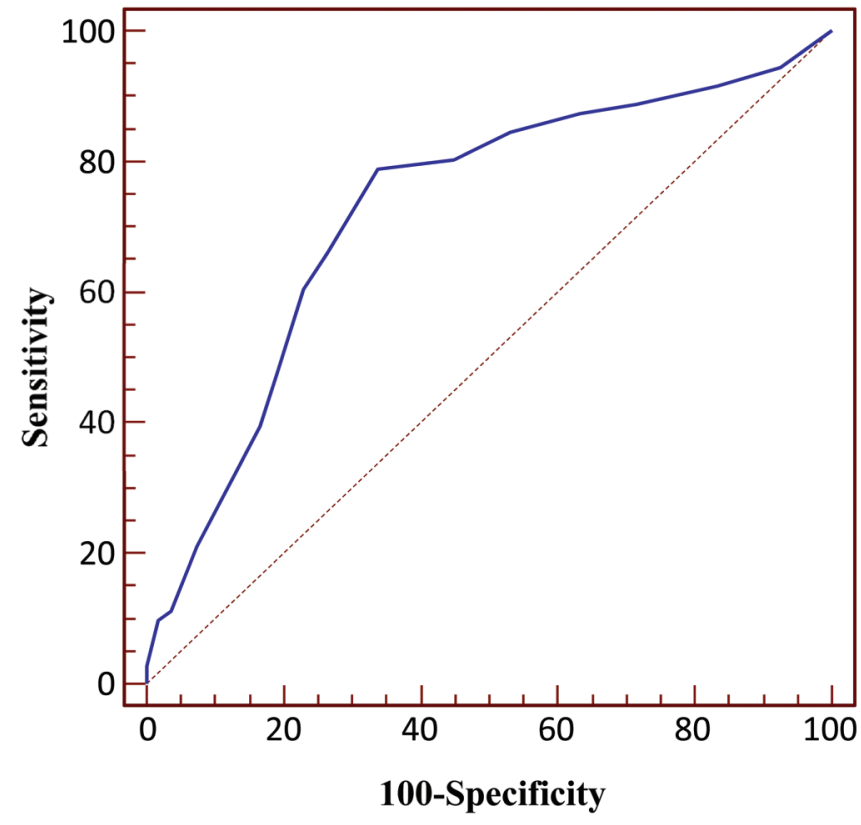

Fig. 2. Receiver operating characteristic for ICT with cutoff value of 14 had sensitivity of $78.87 \%$ and specificity of $66.06 \%$ and an area under the curve value of 0.724 (95\% CI: $0.653-0.788$ ) for diagnosis of MHE.

Abbreviations: $\mathrm{Cl}$, confidence interval; ICT, inhibitory control test; $\mathrm{MHE}$, minimal hepatic encephalopathy. 
Pawar V.B. et al: MHE in cirrhotics

Table 2. Diagnosis of MHE by ICT

\begin{tabular}{lllllll}
\hline MHE BY ICT (Present/Absent) & $n$ & Mean (SD) & $p$-value & Range & CI for mean difference \\
\hline Lures $(\geq 14)$ & Present & 93 & $17.48(2.624)$ & $<0.001$ & $14-24$ & 6.87 to 8.15 \\
& Absent & 87 & $10.01(2.014)$ & & $7-13$ & -19.54 to -16.46 \\
\multirow{2}{*}{ Correct targets } & Present & 93 & $176.54(6.678)$ & $<0.001$ & $162-187$ & $188-200$ \\
& Absent & 87 & $194.54(3.326)$ & & & \\
\hline
\end{tabular}

Abbreviations: CI, confident interval; ICT, inhibitory control test; MHE, minimal hepatic encephalopathy; SD, standard deviation.

not significant $(p=<0.003)$ and did not improve to $<14$ as the cutoff considered in our study. This can be due to the difference in diagnostic methods, as different cutoffs can yield different results.

Lures in patients with PHES were $15.94 \pm 4.18$ and targets were $180.15 \pm 10.20$, while lures in patients without PHES were $17.21 \pm 2.49$ and targets were $177.45 \pm 6.58$ in patients with MHE. The difference between lures in patients with and without PHES positivity was not significant $(p=0.09)$. Similarly, the difference between targets in patients with and without PHES positivity was not significant $(p=0.1463)$.

In CTP class A cirrhosis, MHE reversal was seen in 9/12 patients who were on lactulose, 9/11 patients on rifaximin, and $2 / 10$ patients on placebo. In CTP class $B$ cirrhosis, MHE reversal was seen in $12 / 17$ patients on lactulose, $13 / 20$ patients on rifaximin, and $2 / 18$ patients on placebo treatment. In cases of CTP class C cirrhosis, MHE reversal was seen in 4/6 patients on lactulose, 4/6 patients on rifaximin, and $0 / 8$ patients on placebo. Hence, reversal of MHE was seen in 20/33 (60.6\%) with CTP class A, 27/55 (49.1\%) with CTP class B, and $8 / 20(40 \%)$ with CTP class C ( $p=0.456$ for PHES; $p=0.239$ for ICT). ICT and PHES score did not correlate with severity of liver disease, as measured by CTP or MELD score. A total of 15 patients out of 35 (42.85\%) on lactulose developed some form of side effects, like abdominal bloating and loose motions, which were corrected by adjusting the dose of lactulose; none of the patients from the rifaximin arm or the placebo group had any drug-related side effect $(p=0.0005)$.

Table 3. Results of neuropsychometric testing and ICT in patients with and without MHE

\begin{tabular}{llll}
\hline $\begin{array}{l}\text { Baseline } \\
\text { variables }\end{array}$ & MHE, mean (SD) & & P-value \\
\hline & $\begin{array}{l}\text { Present, } \\
n=108\end{array}$ & $\begin{array}{l}\text { Absent, } \\
n=72\end{array}$ & \\
& $92.29(14.75)$ & $72.88(5.99)$ & $<0.001$ \\
NCT-A & $106.84(24.60)$ & $79.43(8.62)$ & $<0.001$ \\
NCT-B & $90.47(14.83)$ & $75.67(8.01)$ & $<0.001$ \\
SDT & $103.31(20.88)$ & $82.75(9.12)$ & $<0.001$ \\
LTT & $16.47(9.58)$ & $18.07(3.20)$ & $<0.001$ \\
DST & $17.48(2.62)$ & $10.01(2.01)$ & $<0.001$ \\
Lures & $176.54(6.67)$ & $194.54(3.32)$ & $<0.001$ \\
Correct & & & \\
targets & & \multicolumn{2}{c}{} \\
\hline
\end{tabular}

Abbreviations: DST, digit symbol test; LTT, line tracing test; NCT-A, number connection test-A; NCT-B, number connection test-B; SD, standard deviation; SDT, serial dotting test.
We did not follow the patients' serum sodium or ammonia levels, nor take them at baseline, but CTP score and MELD score did not predict MHE positivity in our study.

\section{Discussion}

The diagnosis of MHE is an important health issue. It has significant impact on the HRQOL and ability to drive vehicles, and puts the sufferer at increased risk of developing HE. ${ }^{5-7}$ The prevalence of MHE is variable and has been reported as $30-74 \%$ among patients with liver cirrhosis. ${ }^{3,20}$ In our study, the prevalence of MHE diagnosed through abnormal PHES and/or ICT was $60 \%$. None of our patients had a past episode of overt HE nor did any patient develop an episode of overt HE during the study period. None of our patients had recent alcohol intake, gastrointestinal bleeding, or history of portosystemic shunts, all of which are common risk factors for MHE.

Using the cutoff value of 14 lures, $51.66 \%$ of our patients had MHE by ICT. Using the same cutoff value of 14 lures, MHE was reported by others in $67.5 \%{ }^{23}$ and $40.2 \% .{ }^{23}$ The ICT lures were significantly higher and the response to correct targets was significantly lower in patients with MHE than in those without MHE. The sensitivity of $78.87 \%$ and specificity of $66.06 \%$ with area under the curve of 0.724 (95\% CI: 0.653-0.788) indicates that, in Indian patients, ICT has a much lower sensitivity, specificity, and area under the curve for MHE diagnosis. In one of the previous Indian studies, sensitivity, specificity and receiver operating characteristic of the ICT were $92.6 \%, 78.5 \%$ and 0.855 respectively, ${ }^{22}$ while in another study they were $78 \%, 65.6 \%$ and 0.735 respectively for the diagnosis of MHE in liver cirrhosis. ${ }^{23}$

For the diagnosis of MHE, the PHES is considered to be a reliable tool. It is the preferred tool and has been approved by the working party of the $11^{\text {th }}$ World Congress of Gastroenterology. ${ }^{11}$ The PHES is a battery of paper and pencil tests that relies heavily on the motor function of the patient. The shortcoming of using the PHES for the diagnosis of MHE is that the data needs to be adjusted for the age and education of the person being tested. Secondly, this test can only be administered by a qualified person.

Initially, the studies established a lure threshold of $>5$ as the key variable for the diagnosis of MHE, 7,25 and ICT exhibited a sensitivity and a specificity of $88 \%$ and $77 \%$ respectively for the diagnosis of MHE. This showed good reproducibility. Furthermore, the results of ICT were comparable to those of the PHES. However, it is believed that the lure threshold needs to be adjusted to reflect the local population norms. When compared to the PHES, which examines a wide spectrum of neurocognitive function, ICT examines only attention and response inhibition, but ICT is easier to administer than the PHES. 


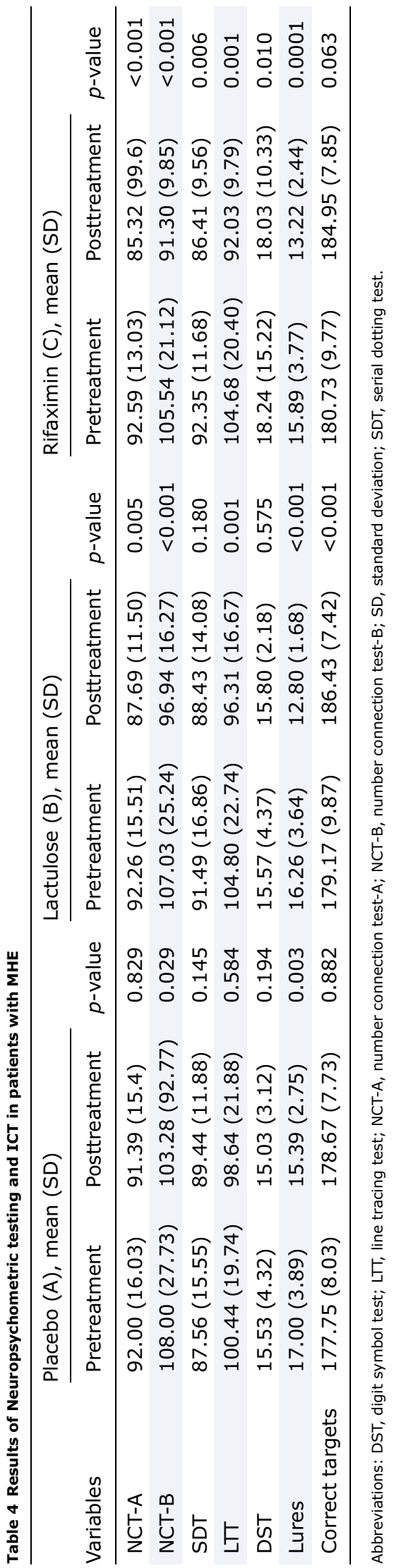

In the current study, the PHES and ICT did not correlate with the severity of liver disease, as measured by CTP score and MELD score. This observation matches results in the previous work done by Taneja et al. ${ }^{23}$ but opposed the findings of Gupta et al. ${ }^{22}$ who found correlation between ICT and severity of liver disease.

Reversal of MHE was seen in 25/35 (71.42\%) patients in the lactulose arm, 26/37 (70.27\%) in the rifaximin arm, and $4 / 36(11.11 \%)$ in the placebo arm $(p<0.001)$. Treatment response to lactulose versus placebo and rifaximin versus placebo both showed statistically significant differences. The treatment responses were almost similar with lactulose and rifaximin. A previous study from a tertiary care center in north India also found that there was reversal in $73.7 \%$ of patients with MHE in the rifaximin arm and $69.1 \%$ in the lactulose arm. ${ }^{26}$ Improvement in MHE was seen in $57 \%$ of patients with lactulose levels at baseline. Low serum sodium and high venous ammonia were highly predictive of nonresponse to lactulose therapy in a study by Sharma et al. ${ }^{27}$

A decrease in the levels of serum endotoxin, proinflammatory markers and arterial ammonia is seen with lactulose treatment. The proinflammatory markers include interleukin-6, interleukin-18, and tumor necrosis factor-alpha. The levels of serum endotoxin, proinflammatory markers and arterial ammonia can be used for the diagnosis of MHE. ${ }^{28}$ Reduction in their levels is associated with improvement in MHE, as evidenced by improvement in PHES and metabolic parameters on magnetic resonance spectroscopy. ${ }^{28}$ Review of eight randomized controlled trials that evaluated lactulose or lactitol for patients who had cirrhosis and HE found that there was no difference between the two forms of treatment. ${ }^{29}$

Rifaximin is a nonsystemic antibiotic. It is, instead, a gutselective antibiotic, which is used to modulate gut flora, reducing levels of bacteria-derived toxins. These toxins are implicated in the pathophysiology of HE. The psychometric performance and HRQOL in patients with MHE is improved by rifaximin. In addition, rifaximin is well tolerated ${ }^{24}$ and easily available in India, costing around INR 190 for 10 tablets. While the cost of rifaximin is almost the same as that of lactulose, it is better tolerated and has no side effects.

There are few other options for the treatment of MHE. These include probiotics and L-ornithine L-aspartate, which have shown reversal of MHE. ${ }^{18}$ Previous studies have demonstrated improvement in HRQOL with the reversal of MHE in patients treated with probiotics. ${ }^{18}$ In the current study, we did not check for improvement of HRQOL.

The inaccessibility of magnetic resonance spectroscopy and electroencephalogram has been the main reason for their scarce use by clinicians in our country for the diagnosis of MHE. Procedures such as magnetic resonance spectroscopy and electroencephalogram are not only operator- but also patient-independent tests. Another technique, the critical flicker frequency ${ }^{25}$ centers on the perception of light as it flickers or fuses as its frequency changes. Sex, level of education and time of day do not significantly affect critical flicker frequency results. However, there is some evidence of differential effects with age and etiology of the underlying liver disease. This testing requires many prerequisites, like intact binocular vision, careful standardization of operating procedures, and optimization of test runs. Moreover, one of the available commercial machines utilizes a red light for the testing, making it is impossible to use for individuals who are red-green color blind. 25 

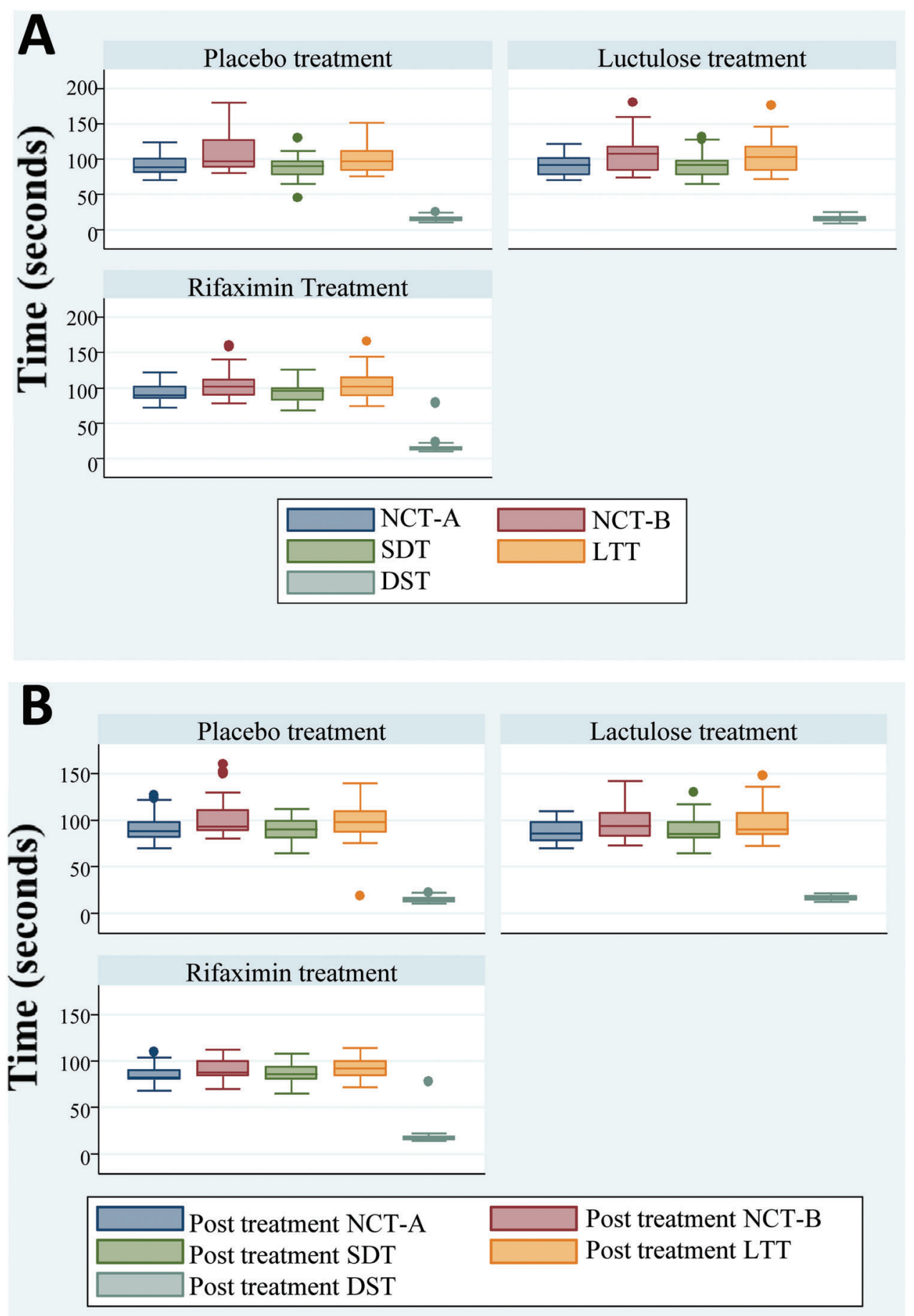

Fig. 3. Box plot showing (A) baseline and (B) posttreatment neuropsychometric results.

Abbreviations: DST, digit symbol test; LTT, line tracing test; NCT-A, number connection test-A; NCT-B, number connection test-B; SDT, serial dotting test.

The primary strengths of our study is that we directly compared PHES and ICT in the diagnosis of MHE and also that we compared the treatment outcomes in patients with MHE using lactulose and rifaximin as treatment. Finding an exact placebo for lactulose is nearly impossible. The most commonly used placebo for lactulose is sorbitol, but it is not an inert placebo and exerts purgative action and purges out ammonia, both of which will affect results (favorably). We used B-complex tablets as placebo to rifaximin, which is readily available and has no purgative action, resulting in minimal confounding. 


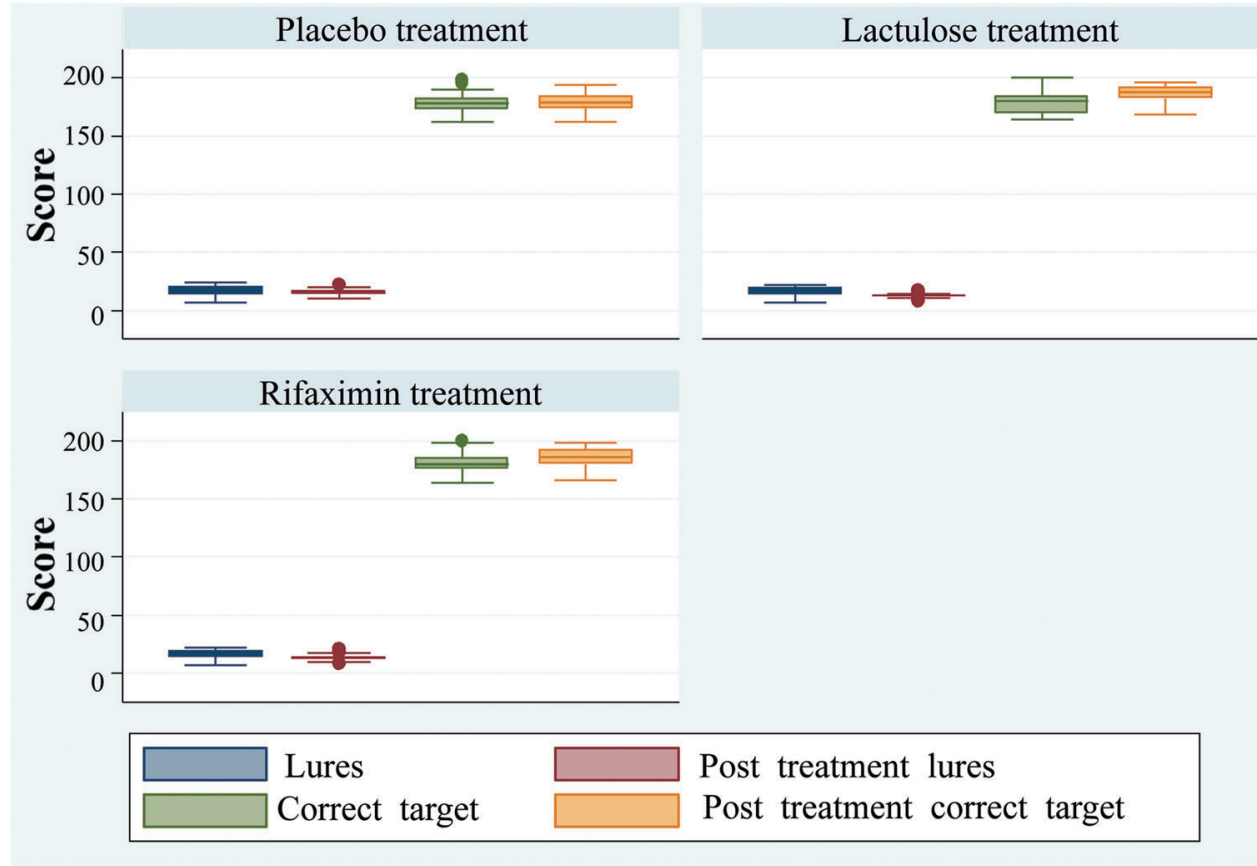

Fig. 4. Box plot showing baseline and posttreatment inhibitory control test results.

The nonresponse to treatment seen in some of our patients may be attributable to other laboratory features, like serum sodium, which we did not looked into. Other reasons may be short duration of treatment, and some might have responded to dual treatment.

ICT is a simple tool for the diagnosis of MHE. However, it has lower sensitivity and specificity in Indian patients. This study evaluated the efficacy of different treatment modalities in MHE in patients presenting at tertiary care center in India. In this group of MHE patients, rifaximin and lactulose were equally efficacious in the treatment of MHE but rifaximin was better tolerated. Hence, we recommend PHES as the primary modality for diagnosis of MHE over ICT in the Indian population. Moreover, the treatment of MHE with rifaximin should be considered over lactulose because of its better tolerability in this set of patients.

It is important to remember that this study was a singlecenter experience and included patients presenting to one of the tertiary care centers in India. Taking into consideration the heterogeneity of the Indian population with respect to genetic makeup, and educational and economic backgrounds, more studies of a similar design will be required at different centers in India in order to accurately extrapolate these results to the entire Indian population. We also recommend further trials based on the design that will include and evaluate efficacy of combination therapies, like rifaximin plus lactulose for treatment of MHE in patients who are nonresponders to monotherapy. Trials looking for recurrence of MHE after stopping treatment could add to our knowledge base and give important inputs regarding follow-up of these patients. Such trials will have ethical implications that must be considered. Moreover, treatment for longer duration may improve efficacy and compliance. Those trials including long duration of the treatment will give further insights to the follow-up of this patient population who undergoes these treatment modalities.

\section{Conflict of interest}

The authors have no conflict of interests related to this publication.

\section{Author contributions}

Conceptualized and designed the study (VBP, NS, VZ, SJ), acquired, analyzed and interpreted the data (VBP, NS), drafted the manuscript (VBP, QC, VZ, SJ), critically revised the manuscript for important intellectual content and supervised the study (VBP, RGS, PMR, QC).

\section{References}

[1] Iacob S, Gheorghe L, Iacob R, Gheorghe C, Hrehoret D, Popescu I. MELD exceptions and new predictive score of death on long waiting lists for liver transplantation. Chirurgia (Bucur) 2009;104:267-273.

[2] Li YY, Nie YQ, Sha WH, Zeng Z, Yang FY, Ping L, et al. Prevalence of subclinical hepatic encephalopathy in cirrhotic patients in China. World J Gastroenterol 2004;10:2397-2401. doi: 10.3748/wjg.v10.i16.2397.

[3] Das A, Dhiman RK, Saraswat VA, Verma M, Naik SR. Prevalence and natural history of subclinical hepatic encephalopathy in cirrhosis. J Gastroenterol Hepatol 2001;16:531-535, doi: 10.1046/j.1440-1746.2001.02487.x.

[4] Romero-Gómez M, Boza F, García-Valdecasas MS, García E, Aguilar-Reina J. Subclinical hepatic encephalopathy predicts the development of overt hepatic encephalopathy. Am J Gastroenterol 2001;96:2718-2723. doi: 10. 1111/j.1572-0241.2001.04130.x.

[5] Prasad S, Dhiman RK, Duseja A, Chawla YK, Sharma A, Agarwal R. Lactulose improves cognitive functions and health-related quality of life in patients with cirrhosis who have minimal hepatic encephalopathy. Hepatology 2007;45: 549-559. doi: 10.1002/hep.21533.

[6] Wein C, Koch H, Popp B, Oehler G, Schauder P. Minimal hepatic encephalopathy impairs fitness to drive. Hepatology 2004;39:739-745. doi: 10 . 1002/hep.20095 
[7] Bajaj JS, Hafeezullah M, Hoffmann RG, Varma RR, Franco J, Binion DG, et al. Navigation skill impairment: Another dimension of the driving difficulties in minimal hepatic encephalopathy. Hepatology 2008;47:596-604. doi: 10 . 1002/hep.22032.

[8] Weissenborn K, Ennen JC, Schomerus H, Rückert N, Hecker H. Neuropsychological characterization of hepatic encephalopathy. J Hepatol 2001;34:768773. doi: 10.1016/S0168-8278(01)00026-5.

[9] Ford JM, Gray M, Whitfield SL, Turken AU, Glover G, Faustman WO, et al. Acquiring and inhibiting prepotent responses in schizophrenia: eventrelated brain potentials and functional magnetic resonance imaging. Arch Gen Psychiatry 2004;61:119-129. doi: 10.1001/archpsyc.61.2.119.

[10] Schiff S, Vallesi A, Mapelli D, Orsato R, Pellegrini A, Umiltà C, et al. Impairment of response inhibition precedes motor alteration in the early stage of liver cirrhosis: a behavioral and electrophysiological study. Metab Brain Dis 2005;20:381-392. doi: 10.1007/s11011-005-7922-4.

[11] Ferenci $P$, Lockwood A, Mullen K, Tarter R, Weissenborn K, Blei AT. Hepatic encephalopathy-definition, nomenclature, diagnosis, and quantification: final report of the working party at the 11th World Congresses of Gastroenterology, Vienna, 1998. Hepatology 2002;35:716-721. doi: 10 1053/jhep.2002.31250.

[12] Epstein JN, Johnson DE, Varia IM, Conners CK. Neuropsychological assessment of response inhibition in adults with ADHD. J Clin Exp Neuropsychol 2001;23:362-371. doi: 10.1076/jcen.23.3.362.1186.

[13] Bajaj JS, Heuman DM, Wade JB, Gibson DP, Saeian K, Wegelin JA, et al. Rifaximin improves driving simulator performance in a randomized trial of patients with minimal hepatic encephalopathy. Gastroenterology $2011 ; 140$ : 478-487.e1. doi: 10.1053/j.gastro.2010.08.061.

[14] Foster KJ, Lin S, Turck CJ. Current and emerging strategies for treating hepatic encephalopathy. Crit Care Nurs Clin North Am 2010;22:341-350. doi: $10.1016 /$ j.ccell.2010.04.007.

[15] Patidar KR, Bajaj JS. Antibiotics for the treatment of hepatic encephalopathy. Metab Brain Dis 2013;28:307-312. doi: 10.1007/s11011-013-9383-5.

[16] Sharma P, Sharma BC. Disaccharides in the treatment of hepatic encephalopathy. Metab Brain Dis 2013;28:313-320. doi: 10.1007/s11011-013-9392-4.

[17] Shukla S, Shukla A, Mehboob S, Guha S. Meta-analysis: the effects of gut flora modulation using prebiotics, probiotics and synbiotics on minimal hepatic encephalopathy. Aliment Pharmacol Ther 2011;33:662-671. doi: 10.1111/j.1365-2036.2010.04574.x.

[18] Mittal VV, Sharma BC, Sharma P, Sarin SK. A randomized controlled tria comparing lactulose, probiotics, and L-ornithine L-aspartate in treatment of minimal hepatic encephalopathy. Eur J Gastroenterol Hepatol 2011;23:725732. doi: 10.1097/MEG.0b013e32834696f5.
[19] Shavakhi A, Hashemi H, Tabesh E, Derakhshan Z, Farzamnia S, Meshkinfar S, et al. Multistrain probiotic and lactulose in the treatment of minimal hepatic encephalopathy. J Res Med Sci 2014;19:703-708.

[20] Dhiman RK, Kurmi R, Thumburu KK, Venkataramarao SH, Agarwal R, Duseja A, et al. Diagnosis and prognostic significance of minimal hepatic encephalopathy in patients with cirrhosis of liver. Dig Dis Sci 2010;55:2381-2390. doi: 10 . 1007/s10620-010-1249-7.

[21] Bajaj JS, Hafeezullah M, Franco J, Varma RR, Hoffmann RG, Knox JF, et al. Inhibitory control test for the diagnosis of minimal hepatic encephalopathy Gastroenterology 2008;135:1591-1600.e1. doi: 10.1053/j.gastro.2008 07.021.

[22] Gupta D, Ingle M, Shah K, Phadke A, Sawant P. Prospective comparative study of inhibitory control test and psychometric hepatic encephalopathy score for diagnosis and prognosis of minimal hepatic encephalopathy in cirrhotic patients in the Indian subcontinent. J Dig Dis 2015;16:400-407. doi: 10.1111/1751-2980.12248.

[23] Taneja S, Dhiman RK, Khatri A, Goyal S, Thumbru KK, Agarwal R, et al. Inhibitory control test for the detection of minimal hepatic encephalopathy in patients with cirrhosis of liver. J Clin Exp Hepatol 2012;2:306-314. doi: 10.1016/j.jceh.2012.07.001.

[24] Sidhu SS, Goyal O, Mishra BP, Sood A, Chhina RS, Soni RK. Rifaximin improves psychometric performance and health-related quality of life in patients with minimal hepatic encephalopathy (the RIME Trial). Am J Gastroenterol 2011;106:307-316. doi: 10.1038/ajg.2010.455.

[25] Morgan MY, Amodio P, Cook NA, Jackson CD, Kircheis G, Lauridsen MM, et al. Qualifying and quantifying minimal hepatic encephalopathy. Metab Brain Dis 2016;31:1217-1229. doi: 10.1007/s11011-015-9726-5.

[26] Sidhu SS, Goyal O, Parker RA, Kishore H, Sood A. Rifaximin vs. lactulose in treatment of minimal hepatic encephalopathy. Liver Int 2016;36:378-385. doi: $10.1111 /$ liv.12921

[27] Sharma P, Sharma BC, Sarin SK. Predictors of nonresponse to lactulose for minimal hepatic encephalopathy in patients with cirrhosis. Liver Int 2009 , 29:1365-1371. doi: 10.1111/j.1478-3231.2009.02067.x.

[28] Jain L, Sharma BC, Srivastava S, Puri SK, Sharma P, Sarin S. Serum endotoxin, inflammatory mediators, and magnetic resonance spectroscopy before and after treatment in patients with minimal hepatic encephalopathy. J Gastroenterol Hepatol 2013;28:1187-1193. doi: 10.1111/jgh.12160.

[29] Gluud LL, Vilstrup H, Morgan MY. Non-absorbable disaccharides versus placebo/no intervention and lactulose versus lactitol for the prevention and treatment of hepatic encephalopathy in people with cirrhosis. Cochrane Database Syst Rev 2016;4:CD003044. doi: 10.1002/14651858.CD003044.pub3. 